\title{
2.2 Important Spectral and Polarized Properties of Semiconducting SWNT Photoluminescence
}

Shigeo Maruyama and Yuhei Miyauchi

\subsubsection{Important Spectral features}

Photoluminescence (PL) of single-walled carbon nanotubes (SWNTs) [1] has been intensively studied for the optical characterization of SWNTs. By plotting PL emission intensities as a function of emission and excitation photon energy, Bachilo et al. [2] obtained a two-dimensional map of relative emission intensities. Hereafter, we refer to such a plot of PL excitation and emission spectra as a "PL map." Figure 1 shows typical PL maps for SWNTs synthesized using different methods. SWNTs were dispersed in $\mathrm{D}_{2} \mathrm{O}$ with surfactant. A major peak in a PL map corresponds to the excitation transition energy of the second subband $\left(E_{22}\right)$ and the photon emission energy of the first subband $\left(E_{11}\right)$ of a specific SWNT structure defined by chiral indices $(n, m)$ [3]. The clear deference between these two PL maps reflects difference of chirality and diameter distribution of SWNTs in different samples. Theoretical studies and recent experiments have clarified that these optical transitions are dominated by strongly correlated electron-hole states in the form of excitons [4-9]. Since the pairs of $E_{11}$ and $E_{22}$ energies depend on the nanotube structure, we can separately measure a photoluminescence excitation (PLE) spectrum from specific $(n, m)$ SWNTs as a cross section of the PL map at an energy corresponding to the emission of the relevant SWNTs. This is useful to study optical properties of different types of SWNTs separately. In addition, as far as semiconducting SWNTs are concerned, such PL mapping is one of the most promising approaches for the determination of the structure distribution in a bulk SWNT sample if complementally combined with other optical spectroscopy such as optical absorption and Raman spectroscopy. Hence, photoluminescence spectroscopy is a powerful tool not only for investigation of physical properties of SWNTs but also in advancing toward development of complete synthesis or separation methods of SWNTs with only one chiral structure, which is one of the ultimate dreams in nano-science and technology field. 
In a PL map, one can generally find some peaks other than bright PL peaks assigned to $E_{22}$ absorption and $E_{11}$ emission of particular nanotube species allowed for incident light polarized parallel to the nanotube axis. Figure 2 shows the typical spectral features of $(7,5)$ SWNTs. First, one can find a broad absorption peak in the shorter excitation wavelength side. Second, one can also find a small absorption peak in the longer emission wavelength side. In addition, for a fixed excitation wavelength, there is a weak emission peak (or peaks) in the longer emission wavelength side. The physical origins of these peaks have been recently demonstrated as, respectively, excitonic phonon sideband in absorption [10-13], absorption peak for perpendicularly polarized light to the nanotube axis $[14,15]$, excitonic phonon sideband in emission $[16,17]$ and/or emission from triplet exciton states $[18,19]$. Recently, there also have been reports on the direct observation of nominally dark excitons for cross-polarized excitons to the nanotube axis (transverse excitons) [20]. Details of these important features will be discussed in the following sections.

Recent development on synthesis techniques of a single nanotube suspended over pillars or trenches enabled one to measure photoluminescence from a "single" SWNT [21]. By single nanotube spectroscopy, further details in the PL and PLE spectra of SWNTs such as excited exciton states [22], effect of surrounding materials on exciton energies (environmental effect) [23-26], temperature dependence of PL homogeneous linewidths [27], and symmetry-induced dark excitons $[28,29]$ have been studied. The observation of excited exciton states gave information about the exciton binding energies in clean, intrinsic SWNTs [22]. Since the surrounding materials change the Coulomb interaction strength through dielectric screening effect, exciton energies depends on surrounding materials around SWNTs [23-26]. The temperature dependence of the homogeneous linewidth has shown almost linear behavior in the low temperature range below $100 \mathrm{~K}$, which suggest that the exciton dephasing is dominated by the interaction between the exciton and the phonon mode with very low energy under lower excitation conditions [27]. Observation of parity-induced dark excitons has been achieved through "brightening" of dark excitons due to symmetry breaking [30] by the Aharonov-Bohm effect [31], which revealed the energy difference of bright and dark excitons about a few to several millielectron volts depending on nanotube diameter 
$[28,29]$.

\subsubsection{Phonon sideband in absorption}

Absorption peaks in PLE spectra about $200 \mathrm{meV}$ above the main absorption/emission peaks of $E_{11}$

[10-13] were attributed to a phonon-assisted excitonic absorption and recombination process [32, 33]. Theoretical prediction of the excitonic phonon sideband shape [33] was in good agreement with the PL spectrum from an individual nanotube [11] and isolated SWNTs in surfactant suspension [12]. These experimental observations of sideband features [10-12] were mainly for the excitation energy range close to the $E_{11}$ transition energy, and the observed sidebands are attributed to excitation to the exciton-phonon bound state. However, the interpretations in these studies were based on only a peak position and lineshape analysis. The direct experimental verification of the origin of the absorption sideband peaks for both $E_{11}$ and $E_{22}$ was achieved by isotope study using SWNTs made of carbon-13 isotope [13].

Figure 3 compares PLE spectra of normal SWNTs and carbon-13 isotope SWNTs (SW ${ }^{13} \mathrm{CNTs}$ ). PLE spectra in Fig. 3 (right panel) correspond to vertical cuts of the PL maps (left panel) at the emission energy indicated by solid lines. Each PLE spectrum is normalized by the $E_{22}$ peak intensity for comparison. It can be seen that the PLE spectra exhibit a sideband 0.2-0.3 eV above the $E_{11}$ and $E_{22}$ main absorption peaks. Since the emission energies of these peaks were almost identical with that at the $E_{22}$ absorption peak, these peaks are also attributed to emission from $(7,5)($ or $(6,5))$ SWNTs. It can be seen from Fig. 3 that the energy difference between the sideband peaks about 0.2 $\mathrm{eV}$ above and the main $E_{22}$ peaks are reduced considerably for the PLE spectra of $\mathrm{SW}^{13} \mathrm{CNTs}$. If these peaks are excitonic phonon sidebands, the amount of the isotope shift is expected to be consistent with the value estimated from the difference of phonon energies. According to theoretical prediction by Perebveinos et. al. [33], the optical phonons near the $K$ point of the graphene Brillouin zone (in-plane TO [16, 34]) have stronger exciton-phonon coupling and are dominantly contribute to 
the sideband. Assuming $K$-momentum phonons are dominant, the isotope shift of the phonon energy is estimated as about $7 \mathrm{meV}$ for considering the square root of mass ratio. Since the energy difference of $\sim 7 \mathrm{meV}$ is in good agreement with the observed energy shift of $6 \sim 10 \mathrm{meV}$ shown in Fig.3, the observed sideband peaks are verified to be excitonic phonon sidebands of the $E_{11}$ and $E_{22}$ main absorption peak due to strong exciton-phonon coupling to the $K$-momentum phonons.

It is seen that the energy differences of these phonon sidebands from the main absorption peaks, $0.2 \sim 0.3 \mathrm{eV}$, are considerably larger than the $K$-momentum optical phonon energy. Here, it should be noted that the energy difference from $E_{22}$ are remarkably similar to the excitonic phonon sideband predicted in Ref. [33]. This larger energy difference of phonon sideband peaks from $E_{11}$ or $E_{22}$ peaks than optical phonon energies can be interpreted by considering phonon-assisted excitation to "dark" (dipole-forbidden) exciton bands by $K$-momontum phonons [33]. Figure 4 shows a schematic diagram of bright and dark excitons in SWNTs [17]. Energy corresponding to circle A in Fig. 4 indicates that the excitation energy of absorption phonon sidebands. Considering the contribution of dark excitons with finite momentum $q_{0}$ (point $\mathrm{X}$ in Fig. 4), the energy contribution from optical absorption required to make a finite- $q_{0}$ exciton is the sum of the energy of a dark exciton and the phonon (or phonons) satisfying energy-momentum conservation. When the dark excitons that dominate the sideband have energies larger than the optically active $E_{\mathrm{ii}}$ exciton, the energy difference between $E_{\mathrm{ii}}$ and the phonon sideband peak can exceed the phonon energy. The amount of contribution of dark exciton band is roughly estimated as about $30-40 \mathrm{meV}$ for $(7,5)$ nanotubes by subtracting phonon energy from the observed energy difference between the $E_{\mathrm{ii}}$ main absorption peaks and the sideband peaks [13]. Recently, energies of $K$-momentum dark excitons have been confirmed by observation of phonon sidebands in emission spectra $[16,17]$. This topic will be discussed in the next section.

\subsubsection{Various sidebands in emission}

In Figure 2, one can find a small sideband feature denoted as "E11-K phonon sideband". This 
sideband was initially reported and explained as "deep dark excitonic states" by Kiowski et al. [35]. They ruled out the possibility of a phonon-dissipating emission mechanism based on their experimental observation that the energy separation between the $E_{11}$ level and the sideband ( $\delta$, see Fig. 4) is remarkably dependent on SWNT diameter $(d)$. On the other hand, Torrens et al. [16] recently proposed that this sideband originates from dipole-forbidden "dark" excitons coupled with $K$-point phonons (i.e., the mechanism that was ruled out in Ref. [35]) based on their experimental measurement of $(6,5)$ SWNTs. Apparently, the dependence of $\delta$ on $d$ is a key issue to resolve this conflict, which is essential for correctly understanding the properties of the weak photoemission found below the $E_{11}$ states.

Recently, Murakami et al. [17] reported that there is no diameter dependence on this sideband. Figure 5 compares PL maps of different SWNTs samples with virtually only one $(n, m)$ structures in each sample. Preparation technique of these samples are presented in Ref. [17]. Panels (d-f) correspond to the same as panels (a-c), but the intensities are presented in logarithmic scale. Panels (d-f) clearly show the existence of sidebands (indicated by the arrows) associated with the PL features of $(6,5),(7,5)$, and $(10,5)$ SWNTs, respectively.

Figures 6 shows PL spectra measured from the three different samples obtained by resonantly exciting $E_{22}$ levels of the dominant SWNT species with excitation wavelengths 570,655 , and 800 $\mathrm{nm}$, respectively. The abscissa denotes energy relative to the PL emission maxima from respective $E_{11}$ levels. Here, spectral decomposition analysis was performed in order to obtain the positions and widths of the observed peaks. Specifically, these spectra were fitted with an $E_{11}$ PL peak, a shoulder at $\sim 45 \mathrm{meV}$ below the $E_{11}$ level and the PL sideband shown in Fig. 5. The shoulder peak at $\sim 45 \mathrm{meV}$ below the $E_{11}$ was also reported in Ref [35] and attributed to another deep dark state. On the other hand, recently, Galland et al. [36] have attributed this peak to the radial breathing mode (RBM) related features. As for Fig. 6(c), additional peaks for $(9,7)$ and $(11,4)$ tubes were taken into account. A 50\% Lorentzian $+50 \%$ Gaussian line shape was assumed for all the peaks. In Fig. 6(a-c), the blue dash curves show the summation of all the components which overlap the experimental spectra shown by the black solid curves. Uncertainties or the possible ranges of the peakmaximum position 
of the sidebands obtained from the decomposition analysis are indicated by horizontal bars. Fig. 6 clearly shows that the observed $\delta$ is independent of $d(\sim 140 \mathrm{meV}$, in the range of $0.75<d<1.05$ $\mathrm{nm})$ within the uncertainties indicated in Figs. 6(a-c). The independence of $\delta$ on $d$ suggests an involvement of phonons in the origin of these sidebands, particularly the involvement of higher-frequency optical phonons that arise from the phonon dispersion relations of graphite [34] and hence is essentially independent of $d$.

Although the above emission sideband peaks whose energy difference from the main $E_{11}$ emission peaks do not depend on the diameter can be attributed to the phonon sideband, Harutyunyan et al. [18] have recently reported that low-energy emission bands can be created in the spectra of SWNTs by intense pulsed laser excitation. The new emission bands appeared $\sim 190 \mathrm{meV}$ below the main $E_{11}$ emission peak of $(6,4)$ SWNTs. This larger energy difference from the main $E_{11}$ emission peak cannot be explained by the phonon assisted mechanism discussed above. From the results of static and time-resolved PL measurements, they attributed this new satellite emission bands to PL from different nominally dark excitons that are "brightened" because of a defect-induced mixing of states with different spins, i.e., intersystem crossing between singlet and triplet exciton states. Here, the dependence of the energy difference between this new satellite emission peaks and the main $E_{11}$ peak on nanotube diameter $d$ is again a key issue. Matsunaga et al. [19] have recently reported that the weak PL peak $\sim 130-140 \mathrm{meV}$ lower than the $E_{11}$ peak diminishes at low temperatures and that the energy separation only weakly depends on the tube diameter, which supports the interpretation that the weak low-energy peak is the phonon sideband of the $K$-momentum dark exciton states as discussed above. In addition to the (emission) phonon sideband, they also found that an additional low-energy PL peak appears under intense pulsed-laser irradiation, and the intensity of which increases with decreasing temperature, and the energy separation depends strongly on the tube diameter. Their results suggest that there can be two kind of origins for satellite peaks around $100-200 \mathrm{meV}$ below the $E_{11}$ peak; one is the $K$-momentum phonon sideband, and another is the spin-induced nominally dark excitons (triplet exciton states). From the above discussion, the apparently complicated diameter dependence of the emission sideband features reported in the 
pioneering work by Kiowski et al. [35] might be attributed to incomplete separation of both sideband features originating from the two different mechanisms (emission from $K$-momentum dark states and triplet dark states). Since intrinsic SWNTs do not have the mechanisms for spin-flip processes, one can expect only the $K$-momentum phonon sideband peaks for intrinsic SWNTs.

\subsubsection{Cross-polarized absorption}

PLE studies of SWNTs have mainly focused on the optical transitions for incident light parallel to the SWNT axis. However, there are also important absorption peaks for cross-polarized light to the nanotube axis. Figure 7 (a) and (b) schematically show the selection rules for incident light polarized parallel and perpendicular to the SWNT axis $[31,37,38]$. In the case of parallel polarization, optical absorption between subbands with the same quasi-angular momentum are allowed ( $\Delta \mu=0$ or $E_{\mathrm{ii}}$ transitions), while $\Delta \mu= \pm 1$ transitions are allowed for perpendicular polarization ( $\mu$ denotes the subband (cutting line) index in the 2D Brillouin zone of graphite). We refer to $\Delta \mu= \pm 1$ transitions between the first and second subbands as $E_{12}$ and $E_{21}$ transitions. Since the emission is primarily caused by recombination of electrons and holes within the first conduction and valence subbands with the same index $\mu$ ( $\Delta \mu=0, E_{11}$ transitions), the emission dipole is considered to be parallel to the SWNT axis. Note that the distinct cross-polarized excitation peaks can be observed although the cross-polarized absorption has been considered to be strongly suppressed and cannot have a peak structure due to the induced self-consistent local field (depolarization effect) within single particle theory [31]. This is one of the most important consequences and signature of the strong Coulomb interaction and excitons in SWNTs.

Optical absorption by two different dipoles being normal to each other followed by the same $E_{11}$ emission process can be separately probed by the polarized PLE spectroscopy on randomly oriented ensemble SWNTs through anisotropy analysis [14]. Use of ensemble sample enables one to probe very weak cross-polarized absorption peaks clearly even without strong light source such as a wavelength variable laser. Figure 7(c) shows the schematic diagram for the measurement of PL 
anisotropies of randomly oriented SWNTs. Because a PL emission signal is enhanced when directions of an emission dipole and the emission polarizer are the same, the direction of emission polarizer determines the mainly probed SWNTs. In the $I_{\mathrm{VV}}$ spectra, the PL emission excited by collinear absorption ( $\Delta \mu=0$ absorption (e.g. $E_{22}$ absorption) $\rightarrow \Delta \mu=0 \quad E_{11}$ emission) is enhanced, whereas emission excited by perpendicular absorption $\left(\Delta \mu= \pm 1\right.$ absorption (e.g. $E_{12}$ absorption) $\rightarrow \Delta \mu=0 \quad E_{11}$ emission) is enhanced in the $I_{\mathrm{VH}}$ spectra. Polarized PLE spectra are obtained with the emission polarizer oriented parallel to $\left(I_{\mathrm{VV}}\right)$ or perpendicular to $\left(I_{\mathrm{VH}}\right)$ the direction of the vertically polarized excitation, and the pure component for parallel $\left(I_{/ /}\right)$and perpendicular $\left(I_{\perp}\right)$ dipoles relative to the nanotube axis were obtained using the relationships $[14,39]$ $I_{/ /}=\left\lfloor\left(r_{\exp }-r_{\perp}\right) /\left(r_{/ /}-r_{\perp}\right)\right\rfloor\left(I_{V V}+2 I_{V H}\right) \quad$ and $\quad I_{\perp}=\left\lfloor\left(r_{/ /}-r_{\text {exp }}\right) /\left(r_{/ /}-r_{\perp}\right)\right\rfloor\left(I_{V V}+2 I_{V H}\right) \quad$, where $r_{\text {exp }} \equiv\left(I_{V V}-I_{V H}\right) /\left(I_{V V}+2 I_{V H}\right)$ is the anisotropy, and $r_{/ /}$and $r_{\perp}$ are the maximum and minimum anisotropies for parallel and perpendicular dipoles, which are related by $r_{\perp}=-0.5 r_{/ /}$[39]. Details of the experimental technique are presented in Ref. [14]. The validity of the spectra obtained by this technique has been confirmed by direct observation of $I_{/ /}$and $I_{\perp}$ spectra by single nanotube spectroscopy [15] and polarized PLE measurements on ensemble SWNTs dispersed in toluene using organic dispersing agent [40].

Figure 8(a) and (b) shows the decomposed PL maps. In Fig.8 (c) and (d) the decomposed PLE spectra of $(7,5)$ and $(6,5)$ SWNTs are presented. For the spectra in Fig. 8, value of $r_{/ /}=0.31$ was determined from the maximum value of observed anisotropy for SWNTs in the lower excitation energy range without any peak structure [14]. While the main peaks corresponding to $\Delta \mu=0$ transitions are seen in the $I_{/ /}$spectra in Fig.8(a) and (c), it can be clearly seen that the $I_{\perp}$ spectra in Fig. 8(b) and (d) exhibit two distinct peaks below the respective $E_{22}$ peaks, and there exist small, but nonzero intensity tails above the distinct peaks in the $I_{\perp}$ maps. The lowest $I_{\perp}$ peaks are relatively sharp, while the second $I_{\perp}$ peaks are broader. Here it was confirmed that the ambiguity of $r_{/ /}$around 0.3 does not substantially change the peak positions of the lowest $I_{\perp}$ peaks. 
One can expect the degeneracy of $E_{12}$ and $E_{21}$, and only one peak around $\sim\left(E_{11}+E_{22}\right) / 2$ assuming electron-hole symmetry in SWNTs. Furthermore, within a single particle theory, one can expect that the $E_{12}$ and $E_{21}$ transitions drop almost in the middle of the $E_{11}$ and $E_{22}$ (i.e., $\left.\sim\left(E_{11}+E_{22}\right) / 2\right)$ [41]. However, the experimental results showed pairs of peaks for cross-polarized absorption bands and the peak energies were considerably blue shifted from $\left(E_{11}+E_{22}\right) / 2$. The prediction from a simple theory cannot reproduce the experimental results. These discrepancy were initially explained as follows [14]: The pairs of cross-polarized absorption peaks were simply attributed to the electron-hole asymmetry in SWNTs (i.e., degeneracy lifting between $E_{12}$ and $E_{21}$ ), or excited exciton states for cross-polarized (transverse) excitons. The higher cross-polarized absorption energies than the expectation from the single particle theory were attributed to smaller exciton binding energies for transverse excitons than excitons polarized parallel to the nanotube axis (longitudinal excitons).

Recent theoretical studies by Uryu and Ando [42, 43], Zhao and Mazumdar [7] and Wang et al. [44] that included electron-electon (e-e) and electron-hole (e-h) Coulomb interactions have shown that the transverse excitons can have finite oscillator strength due to strong excitonic effects, and their excitation energies are considerably blue-shifted from $\left(E_{11}+E_{22}\right) / 2$. As for this point, initial interpretation is virtually correct. However, Wang et al. [44] has reported that only one distinct peak for the cross-polarized absorption is expected because $E_{12}$ and $E_{21}$ excitons interact with each other and one bright and one dark exciton states are created. Considering the mixing of $E_{12}$ and $E_{21}$ excitons, we cannot attribute the pair of peaks simply to the $E_{12}$ and $E_{21}$ excitons. The difference of peak shapes between the two peaks may be a key to address this issue. In order to clarify this issue, further theoretical studies including the electron-hole asymmetry, intervalley mixing, and electron-phonon interactions might be needed.

\subsubsection{Transverse quasi-dark excitons}

As discussed in the previous sections, the optical transitions in semiconducting SWNTs are dominated by strongly bound electron-hole states called excitons [4-8]. Figure 9(a) compares the selection rules for excitation polarized parallel (longitudinal) and perpendicular (transverse) to the 
nanotube axis. Longitudinal excitons consist of electrons and holes in the same 1D subband $\left(E_{11}, E_{22}\right.$, ...). On the other hand, transverse excitons [7, 42] have quasi-angular momentum connecting the electron and hole states across these subbands $\left(E_{12}\right.$ and $\left.E_{21}\right)$. As shown in Fig. 1(b), the degenerate exciton states near the $K$ and $K^{\prime}$ points in momentum space are theoretically predicted to yield optically active (bright) and inactive (dark) exciton states through the intervalley Coulomb interaction for both longitudinal and transverse excitons [7]. Only dark states for longitudinal excitons [28-30] have been experimentally confirmed using the Aharonov-Bohm effect in strong magnetic fields. As for the transverse excitons, if the intrinsic electron-hole asymmetry in SWNTs is large enough to give considerable degeneracy lifting between $E_{12}$ and $E_{21}$ excitons, one can expect that the transverse dark excitons acquire finite oscillator strength even without magnetic field. Hereafter, we refer this nominally dark excitons as transverse quasi-dark excitons.

Recently, we have demonstrated the first direct observation of transverse quasi-dark excitons using polarized photoluminescence excitation spectroscopy [20]. The intrinsic electron-hole (e-h) asymmetry lifts the degeneracy of the transverse exciton wavefunctions at two equivalent $\mathrm{K}$ and $\mathrm{K}^{\prime}$ valleys in momentum space, which gives finite oscillator strength to transverse dark exciton states. The experimental procedure and analysis are almost the same as presented in the previous section, and only differences were the use of wavelength variable laser as a light source, and the use of HiPco SWNTs as a sample. The use of laser and ensemble SWNTs enable us to obtain very weak quasi-dark exciton absorption signals with high sensitivity.

Figure 9(c) and 9(d) shows PLE maps for parallel (longitudinal excitons) and perpendicular (transverse excitons) excitations to the nanotube axis. For perpendicular excitation, the observed PLE peak positions were completely different from those for parallel excitation as discussed in the previous section. Both peak positions and PLE spectral shapes of the dominant peaks for parallel and perpendicular incident light are consistent with those in previous measurements $[2,14,15]$. For parallel excitation, near-infrared PL due to the e-h recombinaiton of longitudinal excitons within the first subband $E_{11}$ by excitation within the second subband $E_{22}$ are observed. On the other hand, $E_{11}$ PL by excitation of transverse excitons between the first and second subbands ( $E_{12}$ and $\left.E_{21}\right)$ is 
observed for perpendicular excitation. The distinct transverse exciton peaks have another broad absorption peaks and intensity tails to the high-energy side, as has been reported previously [14, 15]. Superposition of the $E_{12}$ and $E_{21}$ exciton wavefunctions in the K and K' valleys could produce the bright and dark transverse exciton states as introduced above. Hereafter, we refer the bright transverse exciton states as $E_{12}^{(+)}$.

On the low-energy side of $E_{12}{ }^{(+)}$we found weak but distinct novel absorption peaks approximately 200-300 meV below the $E_{12}{ }^{(+)}$absorption peaks, as shown in the outlined region in Fig. 9(d). We attribute these small absorption peaks below $E_{12}{ }^{(+)}$to exciton absorption by quasi-dark excitons that acquire finite oscillator strength due to the degeneracy lifting of $E_{12}$ and $E_{21}$ excitons originating from the intrinsic e-h asymmetry in SWNTs. We hereafter refer these exciton transitions as $E_{12}{ }^{(-)}$.

Figure 10(a) shows the observed excitation energies for longitudinal and transverse excitons. $E_{12}{ }^{(+)}$ (triangles) and $E_{12}^{(-)}$(squares) were observed between $E_{11}$ and $E_{22}$ (circles). $E_{12}{ }^{(+)}$are close to $E_{22}$, while $E_{12}{ }^{(-)}$are relatively close to $E_{11}$. Note that phonon-related features such as phonon sidebands and Raman scatterings can be ruled out as an explanation for the $E_{12}{ }^{(-)}$peaks because the $E_{12}{ }^{(-)}$peaks do not have constant energy difference from $E_{11}, E_{12}{ }^{(+)}$, and $E_{22}$. Figure 10(b) shows the diameter dependence of the energy difference $\Delta_{12}$ between $E_{12}{ }^{(+)}$and $E_{12}^{(-)}\left(\Delta_{12} \equiv E_{12}^{(+)}-E_{12}^{(-)}\right) . \Delta_{12}$ is approximately 200-300 meV for the observed nanotube species, and depends on the specific nanotube structure. The magnitude of the bright-dark energy splitting for transverse excitons is much larger than that of longitudinal excitons, as is consistent with previous theoretical predictions [7, 43]. Based on comparison between the experimental and theoretical results on the transverse quasi-dark excitons, we can evaluate the degree of e-h asymmetry corresponding to each $(n, m)$ SWNT with specific chiral structures. Details of the chirality dependent e-h asymmetry in SWNTs will be discussed elsewhere [20]. 


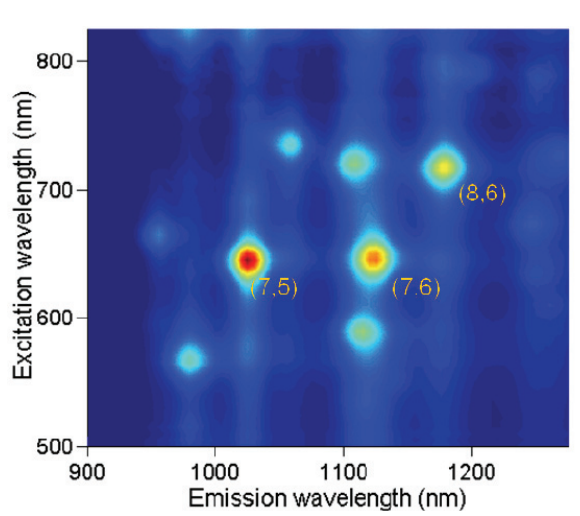

(a) ACCVD $850^{\circ} \mathrm{C}$

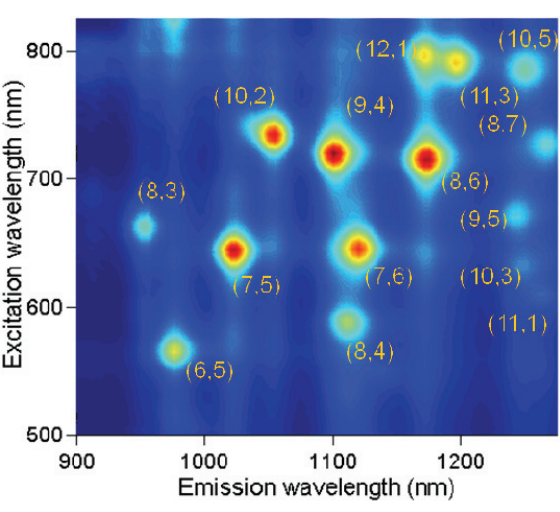

(b) HiPco

Fig 1. PLE map of different samples. (a) Alcohol CCVD (ACCVD) SWNTs and (b) HiPco SWNTs dispersed in $\mathrm{D}_{2} \mathrm{O}$ using surfactant (SDS [1]) [45]. ACCVD SWNTs were produced on zeolite support particles under the conditions of $850{ }^{\circ} \mathrm{C}$ and $10 \mathrm{~min}$ for the CVD temperature and reaction time, respectively.

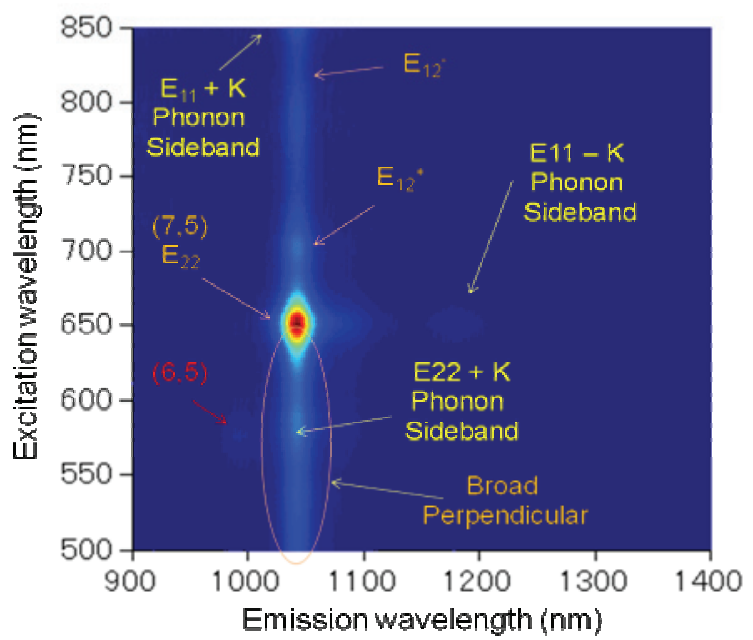

Fig. 2. Spectral features observed in PL map of sample with virtually only $(7,5)$ SWNTs. SWNTs were individually dispersed in toluene using fluorene polymer (PFO [46]). 

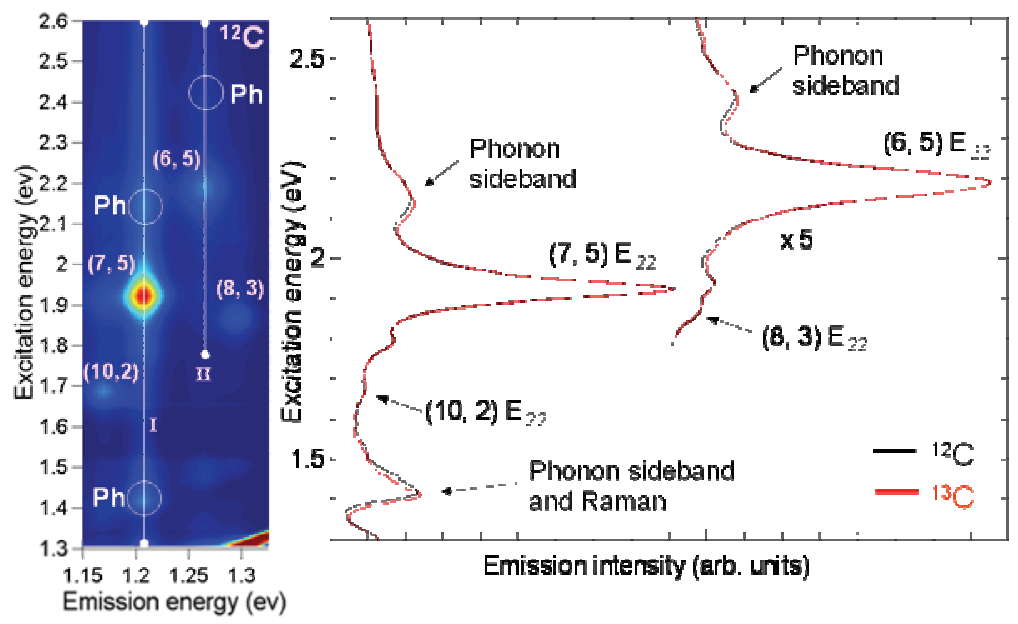

Fig. 3 Phonon sideband for $\mathrm{E}_{11}$ and $\mathrm{E}_{22}$ absorption of $(6,5)$ and $(7,5)$ SWNTs [13].

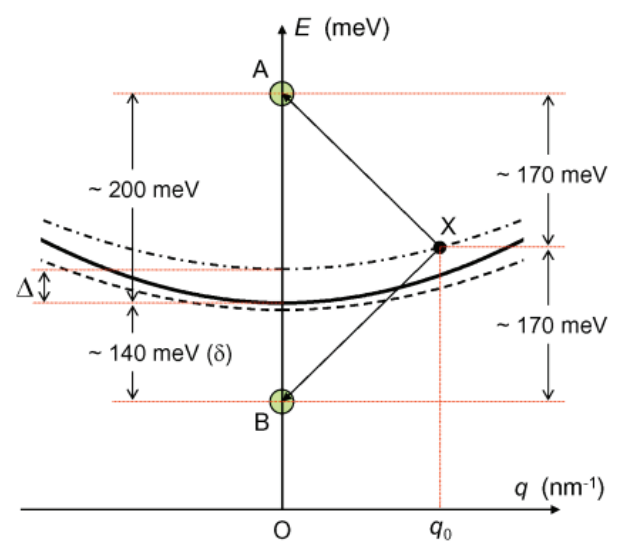

Fig. 4 Schematic diagram showing the four lowest-energy singlet exciton states in zigzag SWNTs [17]. The abscissa denotes the exciton momentum. The solid and dashed curves denote bright and dark exciton bands with zero angular momentum, respectively. The dot-dashed curve denotes the dark exciton band with nonzero angular momentum ( $K$-momentum). 

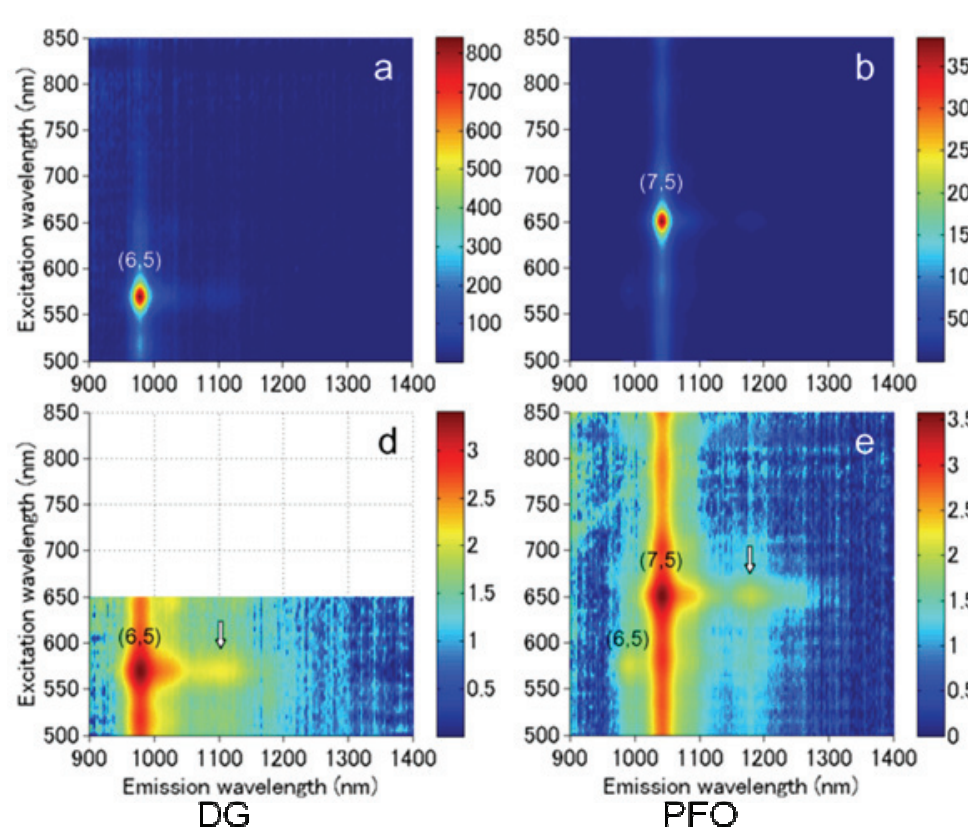

PFO
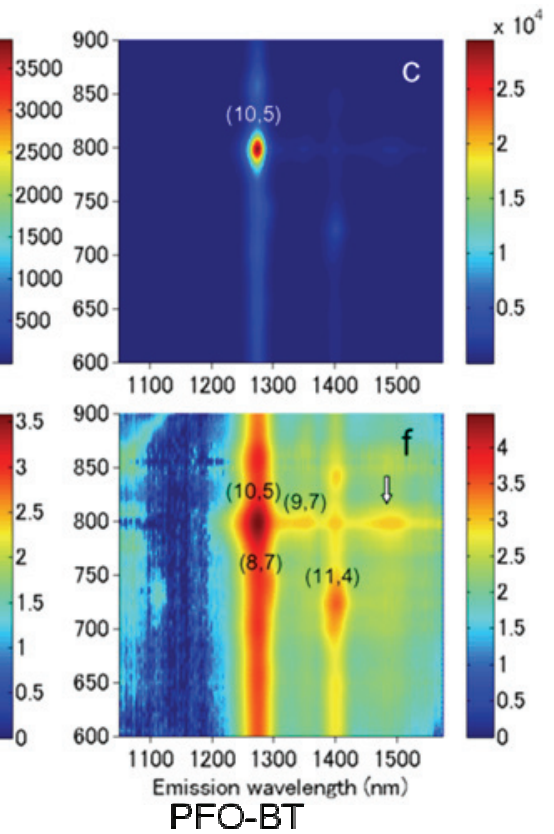

Fig. 5 Contour maps of PL excitation intensities measured from the (a, d) DG, (b, e) PFO, and (c, f) PFO-BT samples, which are dominated by $(6,5),(7,5)$ and $(10,5)$ SWNTs, respectively [17]. Intensity scale-bars are attached to the right of each panel, where the unit is photon count. Panels (d) - (f) are the same as panels (a) - (c), but the intensities are shown in log scale. Arrows indicate the locations of the sidebands. 

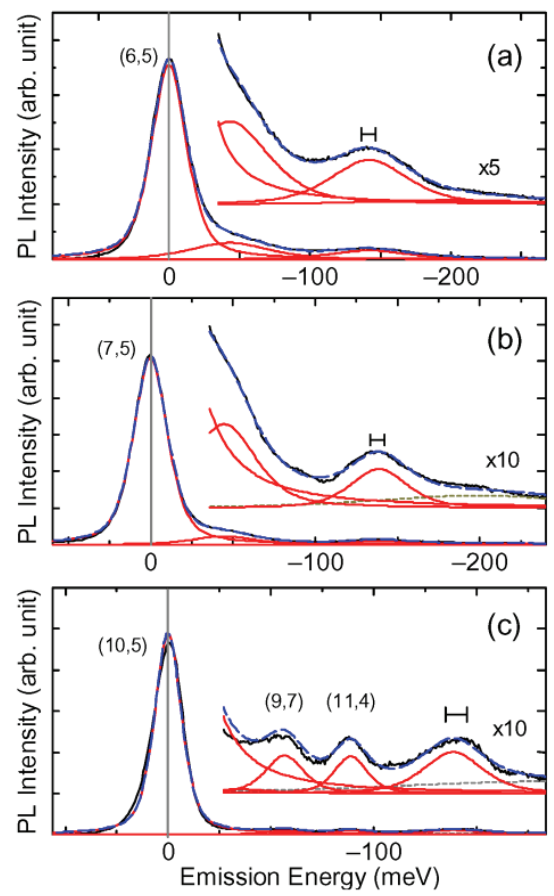

Fig. 6 PL spectra measured for $(6,5),(7,5)$ and $(10,5)$ SWNTs obtained by resonantly exciting the $E_{22}$ levels of the dominant SWNTs with excitation wavelengths of 570, 655, and $800 \mathrm{~nm}$, respectively [17]. The abscissa represents the energy relative to the $E_{11}$ levels of $(6,5),(7,5)$, and $(10,5)$ SWNTs. The original spectra, shown by black solid curves, were decomposed into multiple peaks, and the summation is shown by blue dashed curves. The gray short-dash curves in (b) and (c) are spectral baselines assumed in the analysis. The uncertainties in the peak-maximum positions of the PL sidebands are indicated by the horizontal bars. 


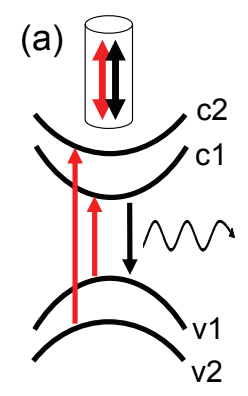

(b) $\underset{v}{\leftrightarrow}$

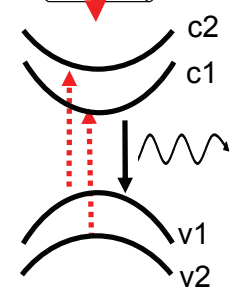

Incident light polarized for z-axis

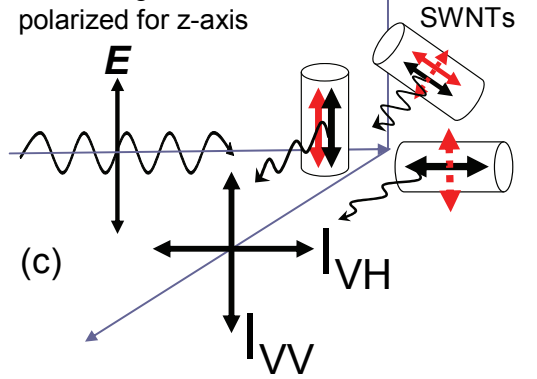

Fig. 7 Schematics of optical transitions in SWNTs corresponding to (a) collinear and (b) perpendicular absorption and emission dipoles [14]. Solid and dotted arrows indicate $\Delta \mu=0$, and $\Delta \mu= \pm 1$ transitions, respectively. (c) Schematic diagram for the measurement of PL anisotropies of randomly oriented SWNTs.
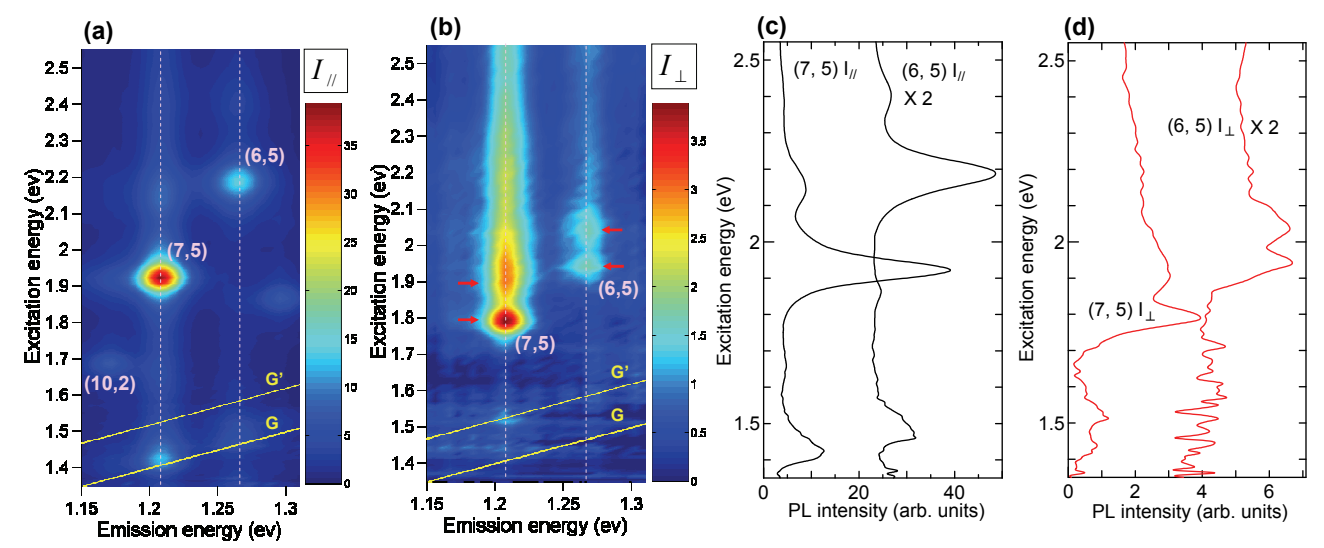

Fig. 8 Decomposed PL maps for (a) collinear $\left(I_{/ /}\right)$and (b) perpendicular $\left(I_{\perp}\right)$ dipoles [14]. Dotted lines in (a) and (b) indicate the emission energies of respective SWNTs. Solid lines indicate the 
position of Raman lines for $\mathrm{G}$ and $\mathrm{G}^{\prime}$ bands. ${ }^{1}$ Peaks for $I_{\perp}$ spectra are indicated by arrows. Decomposed PLE spectra of $(6,5)$ and $(7,5)$ SWNTs for $(c)$ collinear $\left(I_{/ /}\right)$and (d) perpendicular $\left(I_{\perp}\right)$ dipoles. The PLE spectra for $(6,5)$ SWNTs are magnified $(\times 2)$ and offset for comparison in (c) and (d).

(a)
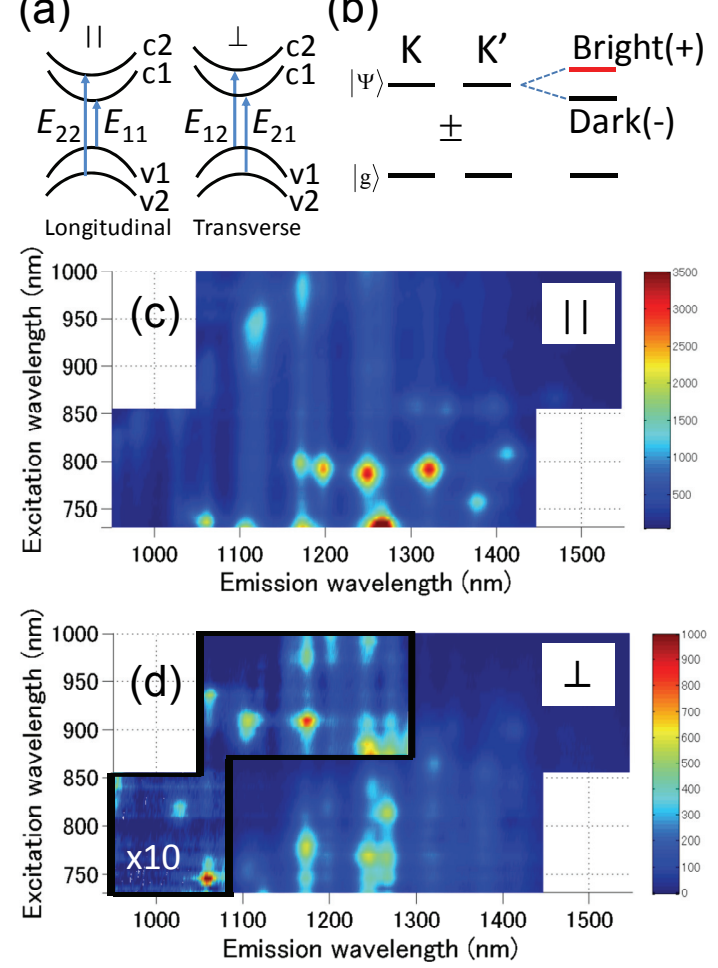

Fig. 9 Schematic diagram of (a) the selection rules for incident light polarized parallel (\|) and perpendicular $(\perp)$ to the nanotube axis, and (b) intervalley mixing of $K$ and $K$ ' excitons [20]. The exciton wavefunctions are even and odd superpositions of those near the $K$ and $K^{\prime}$ points in momentum space. This superposition gives the bright and dark states for longitudinal and transverse excitons. PLE maps for excitations polarized (c) parallel and (d) perpendicular to the nanotube axis. In (d), the PL intensities in the region surrounded by solid lines have been magnified ten times. 


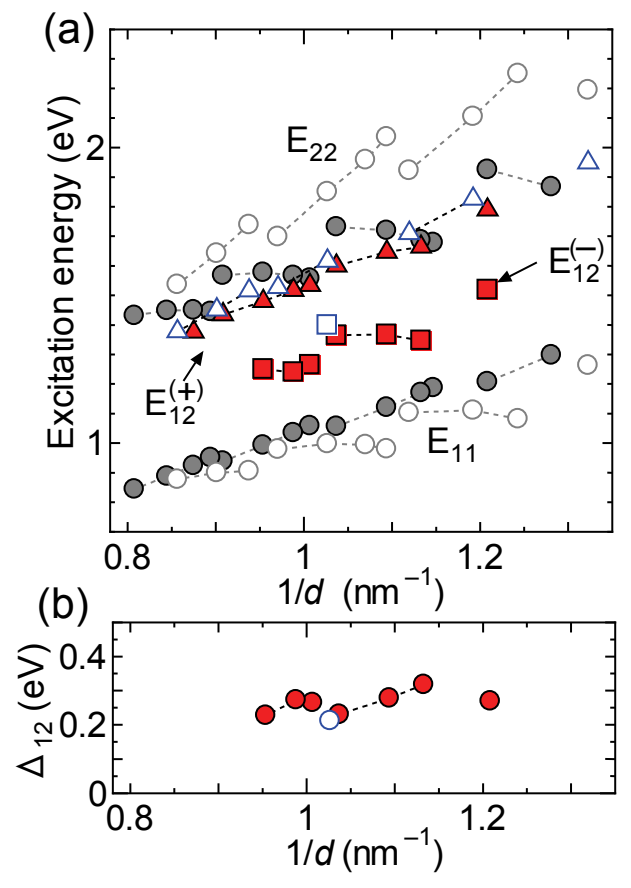

Fig.10 (a) Excitation energy plot for $E_{11}$ and $E_{22}$ (circles), $E_{12}{ }^{(+)}$(triangles), and $E_{12}{ }^{(-)}$(squares) exciton states as a function of inverse diameter [20]. $E_{12}{ }^{(+)}$for $(7,5)$ SWNTs were taken from Ref. [14]. $E_{11}$ and/or $E_{22}$ for small diameter SWNTs were obtained with Xe lamp excitation using a $5 \mathrm{~nm}$ slit width (excitation wavelength below $730 \mathrm{~nm}$ ). (b) Energy difference between $E_{12}{ }^{(+)}$and $E_{12}{ }^{(-)}$ plotted as a function of inverse diameter. In (a) and (b), filled and open marks correspond to type I $(2 n+m \bmod 3=1)$ and type II SWNTs $(2 n+m \bmod 3=2)$, respectively.

\section{References}

[1] M. J. O'Connell et al., Science 297, 593 (2002).

[2] S. M. Bachilo, M. S. Strano, C. Kittrell, R. H. Hauge, R. E. Smalley, and R. B. Weisman, Science 298, 2361 (2002).

[3] R. Saito, G. Dresselhaus, and M. S. Dresselhaus, Physical Properties of Carbon Nanotubes (Imperial College Press, London, 1998).

[4] T. Ando, J. Phys. Soc. Jpn. 66, 1066 (1997).

[5] C. D. Spataru, S. Ismail-Beigi, L. X. Benedict, and S. G. Louie, Phys. Rev. Lett. 92, 077402 (2004).

[6] V. Perebeinos, J. Tersoff, and P. Avouris, Phys. Rev. Lett. 92, 257402 (2004). 
[7] H. Zhao, and S. Mazumdar, Phys. Rev. Lett. 93, 157402 (2004).

[8] F. Wang, G. Dukovic, L. E. Brus, and T. F. Heinz, Science 308, 838 (2005).

[9] J. Maultzsch et al., Phys. Rev. B 72, 241402 (2005).

[10] S. G. Chou et al., Phys. Rev. Lett. 94, 127402 (2005).

[11] H. Htoon, M. J. O'Connell, S. K. Doorn, and V. I. Klimov, Phys. Rev. Lett. 94, 127403 (2005).

[12] F. Plentz, H. B. Ribeiro, A. Jorio, M. S. Strano, and M. A. Pimenta, Phys. Rev. Lett. 95, 247401 (2005).

[13] Y. Miyauchi, and S. Maruyama, Phys. Rev. B 74, 035415 (2006).

[14] Y. Miyauchi, M. Oba, and S. Maruyama, Phys. Rev. B 74, 205440 (2006).

[15] J. Lefebvre, and P. Finnie, Phys. Rev. Lett. 98, 167406 (2007).

[16] O. N. Torrens, M. Zheng, and J. M. Kikkawa, Phys. Rev. Lett. 101, 157401 (2008).

[17] Y. Murakami, B. Lu, S. Kazaoui, N. Minami, T. Okubo, and S. Maruyama, Phys. Rev. B 79, 195407 (2009).

[18] H. Harutyunyan, T. Gokus, A. A. Green, M. C. Hersam, M. Allegrini, and A. Hartschuh, Nano Lett. 9, 2010 (2009).

[19] R. Matsunaga, K. Matsuda, and Y. Kanemitsu, Phys. Rev. B 81, 033401 (2010).

[20] Y. Miyauchi, H. Ajiki, and S. Maruyama, arXiv, 0906.3381 (2009).

[21] J. Lefebvre, J. M. Fraser, P. Finnie, and Y. Homma, Phys. Rev. B 69, 075403 (2004).

[22] J. Lefebvre, and P. Finnie, Nano Lett. 8, 1890 (2008).

[23] S. Chiashi, S. Watanabe, T. Hanashima, and Y. Homma, Nano Lett. 8, 3097 (2008).

[24] Y. Miyauchi, R. Saito, K. Sato, Y. Ohno, S. Iwasaki, T. Mizutani, J. Jiang, and S. Maruyama, Chem. Phys. Lett. 442, 394 (2007).

[25] Y. Ohno, S. Iwasaki, Y. Murakami, S. Kishimoto, S. Maruyama, and T. Mizutani, Phys. Rev. B 73, 235427 (2006).

[26] F. Wang et al., Phys. Rev. Lett. 96, 167401 (2006).

[27] K. Matsuda, T. Inoue, Y. Murakami, S. Maruyama, and Y. Kanemitsu, Phys. Rev. B 77, 033406 (2008).

[28] R. Matsunaga, K. Matsuda, and Y. Kanemitsu, Phys. Rev. Lett. 101, 147404 (2008).

[29] A. Srivastava, H. Htoon, V. I. Klimov, and J. Kono, Phys. Rev. Lett. 101, 087402 (2008).

[30] J. Shaver, J. Kono, O. Portugall, V. Krstic, G. L. J. A. Rikken, Y. Miyauchi, S. Maruyama, and V. Perebeinos, Nano Lett. 7, 1851 (2007).

[31] H. Ajiki, and T. Ando, J. Phys. Soc. Jpn. 62, 1255 (1993).

[32] J. Jiang, R. Saito, A. Gr • eis, S. G. Chou, G. G. Samsonidze, A. Jorio, G. Dresselhaus, and M. S. Dresselhaus, Phys. Rev. B 71, 045417 (2005).

[33] V. Perebeinos, J. Tersoff, and P. Avouris, Phys. Rev. Lett. 94, 027402 (2005). 
[34] R. Saito, A. Jorio, A. G. Souza Filho, G. Dresselhaus, M. S. Dresselhaus, and M. A. Pimenta, Phys. Rev. Lett. 88, 027401 (2002).

[35] O. Kiowski, K. Arnold, S. Lebedkin, F. Hennrich, and M. M. Kappes, Phys. Rev. Lett. 99, 237402 (2007).

[36] C. Galland, A. Högele, H. E. Türeci, and A. Imamoğlu, Phys. Rev. Lett. 101, 067402 (2008).

[37] I. Božović, N. Božović, and M. Damnjanović, Phys. Rev. B 62, 6971 (2000).

[38] A. Grüneis, R. Saito, G. G. Samsonidze, T. Kimura, M. A. Pimenta, A. Jorio, A. G. S. Filho, G. Dresselhaus, and M. S. Dresselhaus, Phys. Rev. B 67, 165402 (2003).

[39] J. R. Lakowicz, Principles of Fluorescence Spectroscopy (Plenum, New York, 1999).

[40] S. Lebedkin, F. Hennrich, O. Kiowski, and M. M. Kappes, Phys. Rev. B 77, 165429 (2008).

[41] A. Grüneis, R. Saito, J. Jiang, G. G. Samsonidze, M. A. Pimenta, A. Jorio, A. G. Souza Filho, G. Dresselhaus, and M. S. Dresselhaus, Chem. Phys. Lett. 387, 301 (2004).

[42] S. Uryu, and T. Ando, Phys. Rev. B 74, 155411 (2006).

[43] S. Uryu, and T. Ando, Phys. Rev. B 76, 115420 (2007).

[44] Z. Wang, H. Zhao, and S. Mazumdar, Phys. Rev. B 76, 115431 (2007).

[45] S. Maruyama, Y. Miyauchi, Y. Murakami, and S. Chiashi, New J. Phys. 5, 149 (2003).

[46] A. Nish, J.-Y. Hwang, J. Doig, and R. J. Nicholas, Nat Nano 2, 640 (2007). 